\title{
Acolhimento da religiosidade/espiritualidade em intervenções psicológicas no hospital: Relato de experiência
}

Embracement of religiosity/spirituality in psychological interventions at the hospital: Experience report

Acogimiento de la religiosidad/espiritualidad en las intervenciones psicológicas en el hospital: Informe de experiencia

\section{Resumo}

Reconhecida como uma dimensão que está associada ao cuidado, a religiosidade/espiritualidade (R/E) vem sendo estudada como um recurso de enfrentamento nos processos de saúde-doença. Contudo, pouco espaço lhe é conferido quando se trata de sua abordagem nos currículos dos cursos das ciências da saúde. Assim, este estudo objetiva descrever como a dimensão da R/E tem sido abordada em intervenções psicológicas no contexto hospitalar. Trata-se de um relato de caso de atendimento desenvolvido com uma paciente com diagnóstico de infecção por HIV/aids em um hospital universitário do interior do Estado de Minas Gerais, Brasil. No caso em questão procurou-se demonstrar como a R/E era vivenciada pela paciente, problematizando os aspectos positivos e negativos dessa dimensão na vida da mesma. Os dados apresentados possibilitam compreender as formas pelas quais a R/E pode ser acolhida pelo psicólogo em sua escuta, alinhando-se aos pressupostos do cuidado integral e humanizado no contexto da saúde pública. Reitera-se a importância do acolhimento da R/E no hospital e a necessidade de maior preparo do profissional para essa prática.

Palavras-chave: Religiosidade; Espiritualidade; Psicologia hospitalar.

\begin{abstract}
Recognized as a dimension that is associated with care, religiosity/spirituality (R/S) has been studied as a coping resource in health-disease processes. However, not much space has been offered in the curricula of health sciences courses. This study aims to describe how the dimension of $\mathrm{R} / \mathrm{S}$ has been addressed in psychological interventions in the hospital context. This is a case report of care developed with a patient diagnosed with HIV/aids in a university hospital in the interior of the state of Minas Gerais, Brazil. In this case, we tried to demonstrate how R/S was experienced by the patient, problematizing the positive and negative aspects of this dimension in her life. The data presented make it possible to understand the ways in which R/S can be welcomed by psychologists in their listening, in line with the assumptions of comprehensive and humanized care in the context of public health. The importance of welcoming the R/S at the hospital and the need for greater preparation of professionals for this practice is reiterated.

Keywords: Religiosity; Spirituality; Hospital psychology.
\end{abstract}

\section{Resumen}

Reconocida como una dimensión asociada al cuidado, la religiosidad/espiritualidad (R/E) ha sido estudiada como un recurso de afrontamiento en los procesos salud-enfermedad. Sin embargo, se le da poco espacio en lo que respecta a 
su enfoque en los planes de estudio de los cursos de ciencias de la salud. Así, este estudio tiene como objetivo describir cómo se ha abordado la dimensión de R/E en las intervenciones psicológicas en el contexto hospitalario. Este es un reporte de caso de atención desarrollado con un paciente diagnosticado con infección por VIH/SIDA en un hospital universitario del interior del estado de Minas Gerais, Brasil. En este caso, intentamos demostrar cómo la paciente vivía la $\mathrm{R} / \mathrm{E}$, problematizando los aspectos positivos y negativos de esta dimensión en su vida. Los datos presentados permiten comprender las formas en que los psicólogos pueden acoger a la R/E en su escucha, en línea con los supuestos de la atención integral y humanizada en el contexto de la salud pública. Se reitera la importancia de acoger a la R/E en el hospital y la necesidad de una mayor preparación de los profesionales para esta práctica.

Palabras clave: Religiosidad; Espiritualidad; Psicología hospitalaria.

\section{Introdução}

As práticas religiosas e espirituais estão presentes em todas as culturas, sendo marcadores sociais que influenciam os modos de vida e as relações estabelecidas entre as pessoas (Rossato, Ullán, \& Scorsolini-Comin, 2021). Essa dimensão faz parte dos contextos de vida da maioria das sociedades e tem atravessado diferentes momentos da construção da humanidade. Deste modo, estas afirmações iniciais nos colocam diante de um primeiro paradoxo: por se tratar de um tema clássico e que atravessa a história do ser humano, como esses conhecimentos têm sido apresentados na contemporaneidade como algo novo, até mesmo inovador? Para respondermos a essa questão é importante discutir de que modo tais dimensões têm sido exploradas no contexto científico e, especificamente, em termos das práticas em saúde.

Observa-se na contemporaneidade um aumento expressivo de pesquisas relacionadas à religiosidade/espiritualidade (R/E) nos contextos de saúde, cuidado e humanização (Cunha, Rossato, \& Scorsolini-Comin, 2021; Cunha \& ScorsoliniComin, 2019; Raddatz, Motta, \& Alminhana, 2019). Religiosidade e espiritualidade são conceitos complexos e distintos. Religiosidade pode ser definida como um sistema de crenças e práticas que se comunicam com o Divino (Sagrado/Deus), compartilhadas por uma comunidade. Já a espiritualidade refere-se a um sentimento pessoal e existencial de busca pelo significado da vida, de uma conexão com o Sagrado. Contudo, como se tratam de conceitos que se sobrepõem em diversos momentos, o termo combinado R/E é comumente encontrado em pesquisas na área da saúde com o propósito de uma discussão mais abrangente (Szaflarski, 2013), com foco nos efeitos dessa dimensão nos desfechos em saúde.

A literatura científica aponta para evidências consistentes de que a R/E exerce um papel significativo em vários aspectos da vida, inclusive na saúde das pessoas e principalmente em sua saúde mental (Koenig et al., 2014; Précoma et al., 2019). Estudos identificaram que a R/E pode atuar como um fator de enfrentamento e adaptação a situações difíceis, contribuindo para a promoção da resiliência, além de que pessoas que vivenciam a R/E têm menos depressão e ansiedade, menos tentativas de autoextermínio, fazem menos uso de substâncias e apresentam melhor qualidade de vida (Brito \& Seidl, 2019; Koenig et al., 2014; Moreira-Almeida, Koenig, \& Lucchetti, 2014; Moreira-Almeida, Lotufo Neto, \& Koenig, 2006; Pargament, Smith, Koenig, \& Perez, 1998). Contudo, Pargament et al. (1998) advertem que a R/E, em algumas ocasiões, pode ser fonte de enfrentamento negativo de um processo de adoecimento, sobretudo quando emerge relacionada a crenças disfuncionais, fanatismo religioso e orienta práticas que não contribuem ou dialogam com os tratamentos formais em saúde.

Em termos de atenção nos contextos de saúde brasileiros, a Política Nacional de Humanização pontua a importância de incorporar a R/E no cuidado aos usuários dos serviços de saúde, além de ter como diretriz o acolhimento nas práticas em saúde. As práticas de acolhimento religiosas e espirituais se tornam essenciais no contexto hospitalar, principalmente em função desse ambiente ser complexo e permeado por múltiplas relações que são atravessadas pelo nascimento, adoecimento, sofrimento, cuidado, atenção, escuta, reabilitação, cura ou morte, fazendo desse local um espaço potencial de desenvolvimento humano que envolve todo o ciclo de vida dos sujeitos. Acolher nesse espaço está relacionado à forma como os usuários são recebidos e atendidos em suas demandas e reais necessidades e principalmente no modo como as relações entre as pessoas acontecem, em como os encontros são produzidos (Brasil, 2013).

Uma das vertentes a ser consideradas pelas equipes de saúde no hospital é a dimensão da R/E. Embora esse 
movimento tenha se intensificado a partir de 1998, em função da ampliação do conceito de saúde pela Organização Mundial da Saúde (OMS), há que se considerar que o cuidado em hospital sempre foi disparador de reflexões acerca da R/E. Assim, podese afirmar que a recomendação da OMS materializou um processo que já emergia nos equipamentos de saúde. No entanto, o aspecto político dessa recomendação tem promovido cada vez mais reflexões sobre práticas que podem ser adotadas, bem como de protocolos para facilitar ao profissional de saúde o acolhimento, de fato, dessa dimensão no cuidar (Précoma et al., 2019).

O processo de adoecimento pode desencadear a procura de significados sobre o propósito da vida e a busca por compreender e dar sentido a uma vivência que pode ser avassaladora, tanto para a pessoa hospitalizada quanto para seus familiares e rede de apoio mais próxima. Neste sentido, as crenças espirituais e religiosas podem oferecer suporte emocional, social e motivação para o tratamento e para a vida, sendo fundamental que o profissional de saúde esteja atento a esta questão (Barbosa, Ferreira, Melo, \& Costa, 2017). Esses aspectos têm sido potencializados a partir da pandemia da COVID-19 a partir de 2020, possibilitando reflexões importantes sobre o sofrimento emocional na população geral e nos profissionais da saúde (Schmidt, Crepaldi, Bolze, Neiva- Silva, \& Demenech, 2020).

Realizar acolhimento, inclusive da R/E dentro do hospital, é papel de todos profissionais de saúde envolvidos no cuidado (Brasil, 2013). Contudo, observa-se uma carência de treinamento na formação desses profissionais para a abordagem dessa dimensão, como exemplificado em estudos realizados no Brasil com estudantes de Medicina e de Enfermagem (Espinha, Camargo, Silva, Pavelqueires, \& Lucchetti, 2013; Lucchetti, Oliveira, Koenig, Leite, \& Lucchetti, 2013).

Ainda no contexto hospitalar destaca-se o papel do psicólogo, profissional que cuida das manifestações da subjetividade humana diante do adoecimento, tais como sentimentos, fantasias, comportamentos, desejos, crenças, conflitos, entre outros. Tais aspectos emocionais repercutem na pessoa hospitalizada, em sua família, em sua rede de apoio afetivo e na equipe de saúde (Simonetti, 2004). É importante que os psicólogos estejam atentos e valorizem a R/E durante sua intervenção, haja vista que sua vivência está relacionada ao processo de saúde/doença, com influências positivas ou negativas na saúde de uma pessoa, podendo atuar como um mecanismo de enfrentamento do sofrimento e de fatores estressantes, mas também levar a emoções consideradas negativas (Cunha \& Scorsolini-Comin, 2019). Contudo, existem desafios para a realização do acolhimento da R/E durante as intervenções psicológicas no contexto hospitalar.

Muitas vezes essa dimensão é negligenciada pelos profissionais de saúde mental pela falta de formação acadêmica e treinamento em como abordar e lidar com esse tema. Além de que muitos profissionais ficam presos em uma ideia de neutralidade científica, no conflito dicotômico entre ciência e religião ou se distanciam ou não conseguem se perceber como pessoas que também vivenciam a R/E (Moreira-Almeida et al., 2014). Esse movimento de afastamento derivado de uma postura de busca pela neutralidade e pelo cientificismo promoveu como efeito, para a formação do profissional de Psicologia, uma dificuldade de entrar em contato com essa dimensão tanto em relação ao usuário, como em relação a si mesmo e a própria prática (Neubern, 2013).

Embora a R/E seja um vértice do cuidado em saúde, ainda permanecem questionamentos e desafios para a atuação do psicólogo no hospital relacionado a esse tema, além de uma lacuna no aprendizado dos profissionais de saúde e de pesquisas sobre o assunto (Raddatz et al., 2019; Scorsolini-Comin, 2018). A partir desse panorama e considerando que os conhecimentos advindos da prática profissional podem disparar reflexões importantes que, posteriormente, também podem ser endereçadas em termos de pesquisas que promovam o avanço do conhecimento em determinado campo, o objetivo deste estudo é apresentar como a dimensão da R/E tem sido abordada em intervenções psicológicas no contexto hospitalar.

\section{Metodologia}

Trata-se de um estudo qualitativo, exploratório, desenvolvido a partir de um relato de experiência profissional que 
utiliza de um estudo de caso. O estudo de caso estudo de caso caracteriza-se como uma ferramenta de pesquisa que permite uma descrição e análise detalhada de algum caso que apresente particularidades e que o torna especial, podendo trazer uma riqueza de dados e informações de modo a contribuir com o saber na área de investigação (Pereira, Shitsuka, Parreira, \& Shitsuka, 2018). O referido estudo foi aprovado pelo Comitê de Ética e Pesquisa da Escola de Enfermagem de Ribeirão Preto da Universidade de São Paulo [Número CAAE: 08064919.7.0000.5393]. Considera-se aqui que os relatos de experiência podem ser importantes ferramentas tanto para nortear os pesquisadores acerca de novas temáticas ou delineamentos e mesmo como um recurso potente para a formação e atualização de profissionais em suas áreas de atuação.

As vivências apresentadas foram extraídas a partir da prática profissional de uma psicóloga ao longo de sete anos de atuação em um hospital universitário de alta complexidade de uma cidade [Informação será inserida após a avaliação]. Este relato foi construído pela descrição de como é realizado o acolhimento religioso por esta profissional em suas intervenções e por reflexões a partir da leitura dos registros de atendimentos realizados nesse período. Tais registros serão apreciados a partir de sua aproximação com a literatura no campo da R/E relacionada à saúde, sobretudo à prática do profissional de Psicologia nesse contexto.

\section{Resultados e Discussão}

As intervenções psicológicas realizadas no hospital, cenário do presente relato, ocorreram a partir de agosto de 2013. O principal objetivo do Serviço de Psicologia desse hospital é promover suporte psicológico à pessoa internada, além de apoio emocional aos seus familiares e a sua rede de apoio socioafetiva, buscando minimizar o sofrimento provocado pelo processo de adoecimento e de hospitalização (Brasil, 2016). Seguindo parâmetros éticos, teóricos e práticos da profissão, cada psicólogo que compõe o serviço tem liberdade para construir sua prática dentro do hospital.

No geral os atendimentos psicológicos são realizados por meio de busca ativa pela psicóloga em sua clínica de referência e/ou mediante solicitação de interconsulta em outras clínicas do hospital. Os atendimentos são registrados no prontuário do usuário. Inicialmente é realizada a leitura do prontuário, de modo a caracterizar dados clínicos e conhecer previamente o histórico de internação. Depois é realizado um primeiro encontro com o usuário e seus familiares, iniciando o processo de acolhimento e de construção do vínculo terapêutico, em que também se verifica se o usuário está em condições clínicas e/ou deseja o atendimento. A partir daí se realiza uma avaliação psicológica.

A avaliação do usuário nesse ambiente tem como objetivo identificar a percepção e a compreensão do processo de hospitalização, do diagnóstico e, quando possível do prognóstico; o sofrimento emocional; o histórico e/ou a presença de alterações psicopatológicas; a adaptação e as reações ao adoecimento e a hospitalização; os recursos de enfrentamento potenciais e existentes; a percepção de si e dos outros; o estilo de vida, as alterações de hábitos de vida; os papéis socioafetivos desempenhados. Além disso, procura-se analisar a dinâmica dos relacionamentos familiares e afetivos; a rede de apoio socioafetiva; as crenças pessoais, religiosas e as relacionadas ao adoecimento e à internação; as expectativas em relação ao processo vivido; as reações emocionais e possíveis estressores; a adesão ao tratamento; o luto por perdas reais e simbólicas e o relacionamento com a equipe e com a instituição (Brasil, 2016).

Esta avaliação psicológica se dá em um processo dinâmico, sendo um espaço também de acolhimento ao usuário e à pessoa que o acompanha. A avaliação pode acontecer em mais de um encontro e na medida em que é realizada também são realizados apontamentos, orientações, mediações com a equipe e oferecimento de suporte psicológico. Conforme as demandas que emergem nesse processo, o usuário e/ou acompanhante seguem em acompanhamento psicológico.

Desde o primeiro encontro, até a avaliação psicológica do usuário e o seguimento do acompanhamento é oferecido um espaço de escuta atenta, permeado pelo respeito, empatia e ausência de julgamentos. É nesse cenário em que se realiza o acolhimento da R/E. Inicialmente busca-se conhecer a afiliação religiosa, bem como práticas e crenças religiosas/espirituais e 
em como essas se relacionam ao processo de adoecimento e de hospitalização e se há o desejo de receber visita religiosa. Mas à medida que o vínculo entre o profissional e o usuário se fortalece, aprofundam-se as questões referentes a essa temática, buscando se compreender o sentido da vida para a pessoa, suas razões para viver, seu relacionamento com o Divino/Sagrado e como essas questões perpassam sua subjetividade e, principalmente, o enfrentamento do processo de saúde-doença, que pode envolver situações complexas, tais como internações prolongadas, no caso de um adoecimento crônico, por exemplo.

Durante esses anos foi possível percerber que a maioria dos usuários atendidos traz a dimensão da R/E durante os atendimentos e grande parte deles diz pertencer a alguma comunidade religiosa, mesmo que não frequentem regularmente uma institução religiosa. Além disso, a maioria dos usuários relata ter crenças espirituais e religiosas, fé e acreditar em Deus sendo manifestadas por exemplo, por meio de orações, leitura de textos religiosos, ao assistirem programas religiosos, pelos símbolos religiosos que levam para o hospital e quando mencionam o desejo de receber visitas religiosas.

É muito comum que usuários e acompanhantes desejarem receber visitas religiosas nesse cenário em tela. No hospital há a Comissão de Assistência Religiosa e Espiritual, que oferece assistência religiosa e espiritual aos usuários, acompanhantes, funcionários e comunidade acadêmica, de acordo com suas crenças e tradições. É esta Comissão que regulamenta e define os horários de visitas religioso-espirituais de acordo com normas do hospital. O hospital também conta com uma capela ecumênica. Destaca-se que esse serviço se baseia nos direitos e garantias individuais e coletivos que são fixados na Constituição Federal de 1988 (Brasil, 1988), que assegura a prestação da assistência religiosa nas entidades hospitalares.

Para ilustrar esse relato apresenta-se o acolhimento da R/E durante o atendimento psicológico junto a uma jovem adulta evangélica, hospitalizada com diagnóstico recente de infecção por HIV/aids e já com infecções associadas à doença. A usuária vinha de um contexto pessoal e familiar bastante religioso, em que o pai era pastor e a jovem participava ativamente da Igreja. Observava-se que a R/E tinha um importante significado na vida da paciente e na vida da família, sendo possível perceber que a mesma encontrava em Deus, em sua fé e em suas práticas religiosas (oração, leitura da Bíblia e nos hinos de louvor) forças e apoio para o enfrentamento das condições de vida que se encontrava.

Para as pessoas vivendo com HIV os aspectos religiosos e espirituais desempenham papéis significativos no processo de enfrentamento, resiliência, adesão e permanência no tratamento. A literatura cientifica têm apontado que essa dimensão psicossocial exerce um papel significativo na adaptação ao diagnóstico, na adesão à Terapia Antirretroviral (TARV), no enfrentamento positivo da infecção e, por consequência, na saúde mental de PVHIV (Szaflarski, 2013).

A jovem relatava com frequência sonhos vívidos que ela avaliava como mensagens de Deus, que tinham grande influência em como ela interpretava e lidava com seu adoecimento e com as intercorrências durante à internação. Nesse contexto, conhecer a R/E da jovem e em como ela perpassava sua vida e de sua família e discutir essa questão junto com a equipe multiprofissional, foi importante para que a equipe oferecesse um cuidado em saúde de qualidade, sem julgamentos e de acordo com a real demanda dessa jovem, pois em um atendimento superficial esses sonhos poderiam ter sido considerados como alucinações e delírios, o que teria impacto na conduta terapêutica dos profissionais.

Durante a internação à jovem iniciou a TARV, valorizando e percebendo a importância do uso desses medicamentos, mas havia a crença na cura da infecção por Deus, tal crença em algum momento poderia levá-la a não adesão aos medicamentos. Essa usuária ainda sentia que seu adoecimento estava relacionado a um castigo Divino, por não ter seguido algumas regras de sua religião, o que lhe trazia bastante angústia. Nesse cenário é importante identificar as crenças limitantes, mas sem agredir a R/E da pessoa, devendo os profissionais de saúde atentarem-se a essa questão de modo a poder reconhecer e prevenir quando a R/E vivenciada como uma fonte de sofrimento e/ou esteja dificultando o tratamento, a adesão, interferindo de modo negativo nas condições emocionais e na qualidade de vida.

No exemplo relatado, a R/E foi experienciada pela paciente como recurso de enfrentamento positivo e negativo, apontando para a complexidade do tema. No geral, a vivência da R/E por parte dos usuários e de seus acompanhantes tem se 
apresentado como uma fonte de suporte e de enfrentamento positivo para lidar com a dor, a angústia, o sofrimento, o processo de adoecimento e de hospitalização. Mas há pessoas que percebem o adoecimento como algo relacionado ao abandono de Deus/do Divino ou a uma forma de punição ou da atuação de forças demoníacas, vivenciando um aumento do sofrimento e do estresse emocional (Pargament et al., 1998).

Assim, o cuidado da R/E nos contextos de saúde deve ser ofertado por profissionais que considerem as crenças dos usuários sem julgá-las, mas também tenham um olhar crítico quando a mesma não é positiva na vida do sujeito ao qual o cuidado está sendo direcionado. Neubern (2013) pontua alguns cuidados ao se abordar a R/E do outro, tais como: não impor a própria R/E ou fazer julgamentos de uma vivência da R/E do outro porque ela se distancia da vivenciada pelo profissional; não abordar superficialmente essa dimensão por insegurança. Além disso, é preciso também perceber se há o desejo da pessoa em falar sobre este tema e não tentar impor essa reflexão, pois nem sempre a pessoa em atendimento quer que essa dimensão seja considerada e isso deve ser respeitado.

Durante os sete anos de trabalho no hospital foi possível perceber que a equipe na maior parte das vezes respeita a vivência da R/E do usuário e que há uma boa receptividade em relação ao acolhimento da R/E. Entretanto, nem sempre esse cuidado é incorporado nas práticas diárias. Geralmente os profissionais que vivenciam sua própria R/E sentem-se mais confiantes e preparados para lidar com a R/E do outro. O trabalho de Nascimento et al. (2013), por exemplo, demonstrou que os enfermeiros reconhecem a articulação entre a religiosidade e a espiritualidade e que a aplicabilidade desses conceitos na prática clínica está relacionada com a vivência da própria $\mathrm{R} / \mathrm{E}$ do profissional, a formação profissional e com o receio das repercussões sobre o papel da R/E na pessoa internada. Para essas autoras é importante promover espaços de discussão sobre o papel da R/E no processo saúde-doença desde o início da formação em Enfermagem e nas ações de educação permanente, para promoção do cuidado integral.

O trabalho no contexto hospitalar permite encontros com uma pluralidade de pessoas, com perfis socioeconômicos, culturais e religiosos múltiplos. De maneira geral a afiliação religiosa nesse contexto é vivenciada como um espaço de acolhimento e suporte social, podendo ser uma facilitadora do vínculo e da retenção nos serviços de saúde (Kisenyi, Muliira, \& Ayebare, 2013).

Para Cunha e Scorsolini-Comin (2019), o acolhimento da R/E precisa ser incorporado na prática profissional dos psicólogos, assim como no currículo dos cursos de Psicologia, com vistas a orientar a formação de novos profissionais e atualizar a prática clínica. Para o acolhimento da R/E o psicológo precisa compreender e valorizar a relevância dessa dimensão na vida e na trajetória da pessoa, inclusive de si próprio. As ações e atitudes mais apropriadas ao se abordar a R/E no contexto clínico são o respeito; saber separar o que é do profissional e o que é do outro; saber ouvir; questionar o papel da R/E na vida da pessoa em atendimento; conhecer sobre o assunto e não impor o próprio conhecimento, práticas e/ou doutrina.

Entretanto, permanecem vários desafios para o acolhimento da R/E no contexto de saúde, pois se sabe que ainda existem muito preconceito e receios em relação à incorporação da R/E no processo terapêutico. Há na Psicologia uma diversidade de referenciais teórico-práticos, com posicionamentos distintos em relação à prática clínica, que trazem discussões e divergências sobre a neutralidade do psicoterapeuta, que nem sempre é bem compreendida, podendo causar controvérsias e confrontos de opiniões com relação à temática (Cunha \& Scorsolini-Comin, 2019). Existem também as discussões em relação à laicidade do Estado brasileiro. Assim, pontua-se que uma abordagem laica em saúde não significa não reconhecer o direito do outro em manifestar sua R/E, mas sim acolher com respeito e sem julgamentos todas as formas de vivenciar a R/E, inclusive quando o usuário manifesta não possuir uma R/E ou desejar que essa dimensão não seja considerada no processo do cuidado.

\section{Considerações Finais}

Por meio de nossas experiências profissionais e com base nas publicações encontradas na literatura científica tem 
ficado evidente que a dimensão da R/E está intimamente relacionada ao processo saúde-doença-cuidado. Contudo, ainda permanecem questionamentos e desafios em relação à abordagem dessa dimensão no contexto hospitalar. A R/E é um vértice do cuidado em saúde, mas por vezes acaba ficando em segundo plano, sendo importante incorporar essa dimensão na prática clínica cotidiana, fomentar pesquisas com essa temática e abrir espaços para o compartilhamento de relatos de experiência profissional que estimulem o cuidado integral e humanizado em saúde, que contemplem o ser humano em todas as suas dimensões.

Acolher a dimensão da R/E é considerar a pessoa para além da queixa. Para tal é importante que aconteça um encontro verdadeiro entre o profissional e o usuário do serviço de saúde. É importante aceitar a narrativa da mística do outro, sem que o profissional a contraponha com sua própria R/E. Para isso é necessário reconhecer qual R/E é a sua e qual é a R/E da pessoa em atendimento, sendo que a atuação do profissional deve permitir que a R/E do usuário possa vir à tona.

Considera-se que na atualidade ainda permanece uma lacuna na formação dos profissionais de saúde, principalmente dos psicólogos, em relação à R/E, sendo preciso integrar essa dimensão nos currículos e nos cursos de formação continuada. Reitera-se que os profissionais de saúde devem valorizar e estar atentos a essa dimensão em sua prática diária.

Este estudo reforça a necessidade de que a R/E seja considerada na formação no ensino superior e técnico de cursos que estejam voltados para a atenção e cuidado à saúde, uma vez que essa dimensão está presente no contexto sociocultural brasileiro e interfere na subjetividade, comportamentos e modos de vida da população. Do mesmo modo os dados aqui apresentados podem servir de subsídios para que profissionais que já estejam inseridos em contextos de atuação possam utilizar deste material como um recurso para refletirem sobre as práticas de atendimento que desenvolvem junto a seus pacientes.

Cabe destacar também que as reflexões apresentadas nesse manuscrito permitem considerar a R/E como uma dimensão que interfere na vida dos sujeitos e nas condições de saúde mental de forma positiva e negativa, devendo ser observada com cuidado, considerando as particularidades de cada vivência dos pacientes e também de seus familiares. O cuidado religioso e espiritual nos contextos de atenção à saúde precisa considerar as vivências religiosas e espirituais dos pacientes como algo que na maioria das vezes é inerente aos contextos de vida. Assim, pode-se proporcionar uma atenção integral e humanizada para pessoas que, na maioria das vezes, estão em uma condição de fragilidade emocional e de saúde física.

As evidências apresentadas na literatura científica, bem como os documentos e informes disponibilizados por organizações internacionais que tem por enfoque o cuidado em saúde, fornecem subsídios para compreender a importância da R/E nas práticas multiprofissionais. Do mesmo modo, compreender os aspectos positivos e negativos dessa dimensão na vida dos sujeitos, grupos e instituições por meio de estudos científicos mostra-se pertinente, para o oferecimento de subsídios para a implementação de novas práticas humanizadas de cuidado. Pesquisas futuras que procurem compreender o papel e as influências dos aspectos religiosos e espirituais na subjetividade, nos comportamentos, na organização social, nos aspectos morais e culturais são profícuos e necessários de serem implementadas.

\section{Agradecimentos}

Os autores agradecem ao Hospital de Clínicas da Universidade Federal do Triângulo Mineiro e à Escola de Enfermagem de Ribeirão Preto da Universidade de São Paulo pelo apoio institucional; à Coordenação de Aperfeiçoamento de Pessoal de Nível Superior (Capes) pela concessão de duas bolsas de doutorado da segunda autora e do terceiro autor; e ao Conselho Nacional de Desenvolvimento Científico e Tecnológico (CNPq) pela bolsa produtividade concedida ao quarto autor. 


\section{Referências}

Barbosa, R. M. D. M., Ferreira, J. L. P., Melo, M. C. B. D., \& Costa, J. M. (2017). A espiritualidade como estratégia de enfrentamento para familiares de pacientes adultos em cuidados paliativos. Revista da SBPH, 20(1), 165-182. http://pepsic.bvsalud.org/scielo.php?script=sci_arttext\&pid=S151608582017000100010

Brasil. (1988). Constituição da República Federativa do Brasil. Presidência da República. Casa Civil. Subchefia para Assuntos Jurídicos. Brasília: Autor. http://www.planalto.gov.br/ccivil_03/constituicao/constituicao.htm

Brasil. (2013). Cadernos Humaniza SUS. Ministério da Saúde. Secretaria de Atenção à Saúde. Política Nacional de Normalização. Departamento de Ações programáticas e Estratégicas. Brasília: Autor. http://bvs.saude.gov.br/bvs/publicacoes/cadernos_humanizaSUS.pdf

Brasil. (2016). Protocolo Operacional Padrão Psicologia - Unidade Psicossocial do HC- UFTM. Ministério da Educação. Hospital de Clínicas do Triângulo Mineiro. Empresa Brasileira de Serviços Hospitalares. Autor.

Brito, H. L. D., \& Seidl, E. M. F. (2019). Resilience of people with HIV/AIDS: Influence of religious coping. Trends in Psychology, 27(3), 647-660. https://doi.org/10.9788/TP2019.3-04

Cunha, V., Rossato, L., \& Scorsolini-Comin, F. (2021). Religião, religiosidade, espiritualidade, ancestralidade: Tensões e potencialidades no campo da saúde. Revista Relegens Thréskeia, 10(1), 143-170. http://dx.doi.org/10.5380/rt.v10i1.79730

Cunha, V. F., \& Scorsolini-Comin, F. (2019). Best professional practices when approaching religiosity/spirituality in psychotherapy in Brazil. Counselling and Psychotherapy Research, 19(4), 523-532. https://doi.org/10.1002/capr.12241

Espinha, D. C. M., Camargo, S. M., Silva, S. P. Z., Pavelqueires, S., \& Lucchetti, G. (2013). Opinião dos estudantes de enfermagem sobre saúde, espiritualidade e religiosidade. Revista Gaúcha de Enfermagem, 34(4), 98-106. https://doi.org/10.1590/S1983-14472013000400013

Kisenyi, R. N., Muliira, J. K., \& Ayebare, E. (2013). Religiosity and adherence to antiretroviral therapy among patients attending a public hospital-based HIV/AIDS clinic in Uganda. Journal of Religion and Health, 52(1), 307-317. https://doi.org/10.1007/s10943-011-9473-9

Koenig, H. G., et al. (2014). Religious involvement is associated with greater purpose, optimism, generosity and gratitude in persons with major depression and chronic medical illness. Journal of Psychosomatic Research, 77, 135-143. https://doi.org/10.1016/j.jpsychores.2014.05.002

Lucchetti, G., Oliveira, L. R., Koenig, H. G., Leite, J. R., \& Lucchetti, A. L. (2013). Medical students, spirituality and religiosity-results from the multicenter study SBRAME. BMC Medical Education, 13, 162. https://doi.org/10.1186/1472-6920-13-162

Moreira-Almeida, A., Koenig, H. G., \& Lucchetti, G. (2014). Clinical implications of spirituality to mental health: Review of evidence and practical guidelines. Brazilian Journal of Psychiatry, 36(2), 176-182. https://doi.org/10.1590/1516-4446-2013-1255

Moreira-Almeida, A., Lotufo Neto, F., \& Koenig, H. G. (2006). Religiosidade e saúde mental: Uma revisão. Brazilian Journal of Psychiatry, 28(3), 242-250. https://doi.org/10.1590/S1516-44462006005000006

Nascimento, L. C., et al. (2013). Espiritualidade e religiosidade na perspectiva de enfermeiros. Texto \& Contexto Enfermagem, 22(1), 52- 60. https://doi.org/10.1590/S0104-07072013000100007

Neubern, M. S. (2013). O que significa acolher a espiritualidade do outro? Considerações de uma clínica etnopsy. In M. H. Freitas, G. J. Paiva, \& C. C. Moraes (Orgs.), Psicologia da religião no mundo ocidental contemporâneo: Desafios da interdisciplinaridade - Volume II (pp. 145-183). EDUCB.

Pargament, K., Smith, B., Koenig, H., \& Perez, L. (1998). Patternsof positive and negative religious coping with major life stressors. Journal for the Scientific Study of Religion, 37, 710-724. https://doi.org/10.2307/1388152

Pereira, A. S., Shitsuka, D. M., Parreira, F. J., \& Shitsuka, R. (2018). Metodologia da pesquisa científica. $1^{\text {a }}$ Ed. Santa Maria: UFSM. Disponível em: http://repositorio.ufsm.br/handle/1/15824

Précoma, D. B., et al. (2019). Atualização da diretriz de prevenção cardiovascular da Sociedade Brasileira de Cardiologia - 2019. Arquivos Brasileiros de Cardiologia, ahead print, 0-0. http://www.dx.doi.org/10.5935/abc.20190204

Raddatz, J. S., Motta, R. F., \& Alminhana, L. O. (2019). Religiosidade/espiritualidade na prática clínica: Círculo vicioso entre demanda e ausência de treinamento. Psico-USF, 24(4), 699-709. https://doi.org/10.1590/1413-82712019240408

Rossato, L., Ullán, A. M., \& Scorsolini-Comin, F. (2021) Religious and spiritual practices used by children and adolescents to cope with cancer. Journal of Religion and Health. https://doi.org/10.1007/s10943-021-01256-Z

Scorsolini-Comin, F. (2018). A religiosidade/espiritualidade no campo da saúde. Revista Ciências em Saúde, 8, 1-2. https://doi.org/10.21876/rcsfmit.v8i2.752

Schmidt, B., Crepaldi, M. A., Bolze, S. D. A., Neiva-Silva, L., \& Demenech, L. M. (2020). Saúde mental e intervenções psicológicas diante da pandemia do novo Coronavírus (COVID-19). Estudos de Psicologia (Campinas), 37, e200063. https://doi.org/10.1590/1982-0275202037e200063

Simonetti, A. (2004). Manual de psicologia hospitalar. Casa do Psicólogo.

Szaflarski, M. (2013). Spirituality and religion among HIV-infected individuals. Current HIV/AIDS Reports, 10(4), 324-332. https://doi.org/10.1007/s11904013-0175-7 